\title{
Novel Techniques of Single-Carrier Frequency-Domain Equalization for Optical Wireless Communications
}

\author{
Kodzovi Acolatse, ${ }^{1}$ Yeheskel Bar-Ness, ${ }^{1}$ and Sarah Kate Wilson ${ }^{2}$ \\ ${ }^{1}$ Department of Electrical and Computer Engineering, New Jersey Institute of Technology, Newark, NJ 07102, USA \\ ${ }^{2}$ Department of Electrical Engineering, Santa Clara University, Santa Clara, CA 95053, USA
}

Correspondence should be addressed to Kodzovi Acolatse, ka2@njit.edu

Received 16 April 2010; Revised 29 July 2010; Accepted 26 September 2010

Academic Editor: Naofal Al-Dhahir

Copyright ( $(2011$ Kodzovi Acolatse et al. This is an open access article distributed under the Creative Commons Attribution License, which permits unrestricted use, distribution, and reproduction in any medium, provided the original work is properly cited.

\begin{abstract}
We investigate the use of single carrier frequency domain equalization (SCFDE) over a diffuse optical wireless (DOW) communications. Recently orthogonal frequency division multiplexing (OFDM) has been applied to DOW communications. However, due to high peak-to-average power ratio (PAPR), the performance of OFDM can severely be affected by the nonlinear characteristics of light emitting diodes (LED). To avoid a PAPR problem, we present in this paper a modified form of SCFDE for DOW communications. We propose three different ways of using SCFDE with DOW communications and show that they exhibit lower PAPR and provide better bit-error rate (BER) performance in the presence of the LED nonlinearity.
\end{abstract}

\section{Introduction}

Due the increase in the number of portable information terminals in work and at home, the demand for highspeed indoor wireless communication has been growing. Recently, the optical spectrum which has virtually unlimited bandwidth has been receiving growing interest for use in indoor wireless data transmission $[1,2]$. Diffuse optical wireless (DOW) communications offer a viable alternative to radio frequency $(\mathrm{RF})$ communication for indoor use and other applications where high performance links are needed. RF systems can support only limited bandwidth because of restricted spectrum availability and interference while this restriction does not apply to DOW links. In indoor DOW systems, light emitting diodes (LED) are used as transmitters and photo-diodes as the receivers for optical signals. These opto electronic devices are cheaper as compared to RF equipments.

Orthogonal frequency division multiplexing (OFDM) modulation is a promising modulation scheme for indoor DOW communication [3-8]. It offers high data rate and high bandwidth efficiency capabilities and provides a means to combat inter-symbol-interference (ISI) that results from multipath propagation. Among the OFDM systems for DOW transmission, the asymmetrically clipped optical orthogonal frequency division multiplexing (ACO-OFDM) [7] has been shown to be more efficient in terms of optical power than the systems that use DC-biased [9]. ACO-OFDM is a form of OFDM that modulates the intensity of an LED. Because ACO-OFDM modulation employs intensity modulation and direct detection (IM/DD), the time-domain transmitted signal must be real and positive. The block diagram of an IM/DD DOW system is depicted in Figure 1. To ensure a real signal, ACO-OFDM subcarriers have Hermitian symmetry, and to obtain a positive signal, only the odd subcarriers are modulated by the data and any time-domain negative values are clipped at the transmitter. It is shown in [7] that the clipping does not distort the data on the odd subcarriers but does reduce the amplitude of their constellation values by a half. The clipping noise is added only to the even subcarriers. The data symbols can be easily detected by demodulating only the odd subcarriers. However, ACO-OFDM signals, like other OFDM systems, have inherently high PAPR, hence its performance can potentially be severely affected by the nonlinear behavior of the LED $[10,11]$. For this reason, single carrier with frequency domain equalization systems have been proposed in optical communication as an alternative to OFDM $[12,13]$. In [12], single carrier frequency domain 


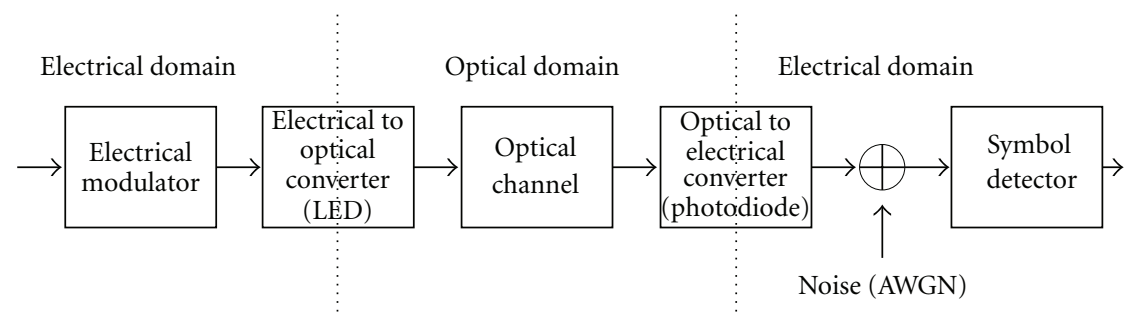

FIGURE 1: Block diagram of intensity modulated/direct detection (IM/DD) DOW communication system.

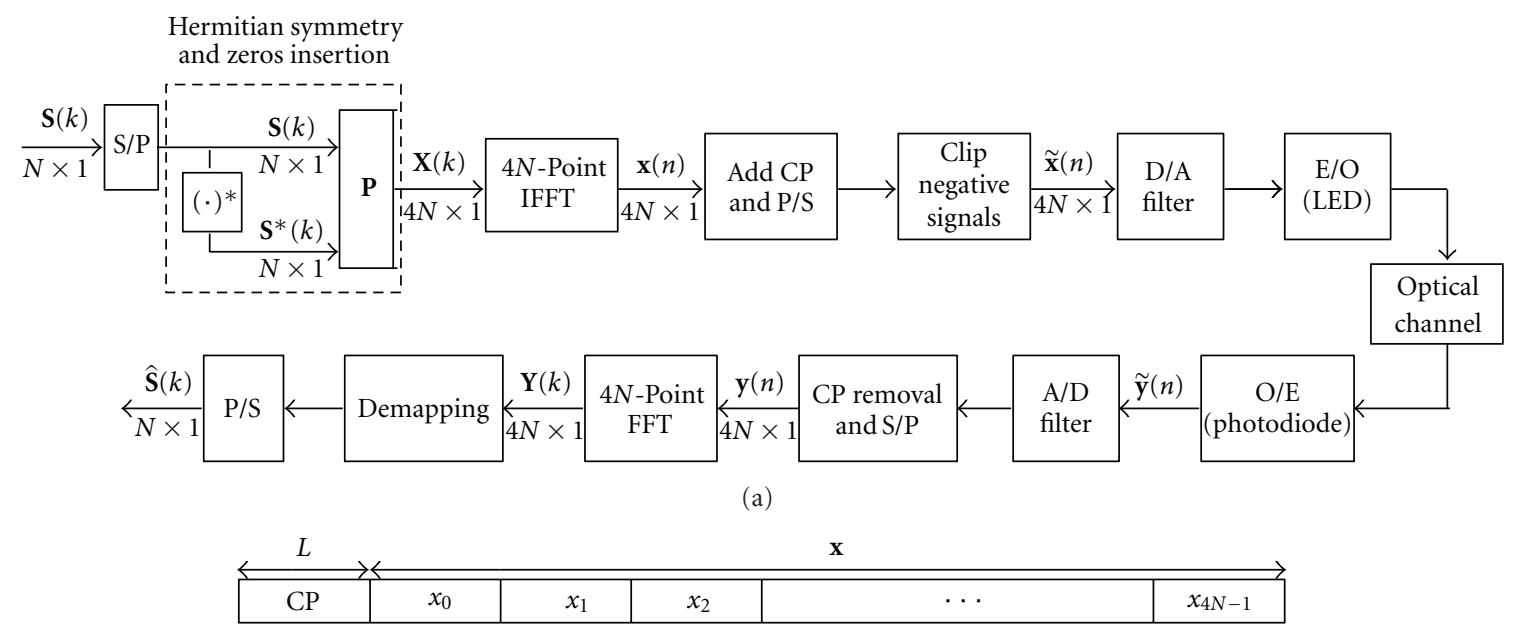

(b)

FIGURE 2: (a) ACO-OFDM transmitter and receiver configuration. (b) ACO-OFDM symbol after cyclic extension.

equalization (SCFDE) signal is transmitted over an optical fiber with coherent detection while SCFDE is combined with pulse position modulation (PPM) in [13] for IM/DD DOW transmission. SCFDE applied with coherent detection has also been presented in [3]. In this paper, we suggest applying the concept of asymmetric clipping of [7] to SCFDE which we denote ACO-SCFDE for IM/DD transmission over a DOW channel.

Single-carrier modulation using frequency domain equalization is a promising alternative to OFDM for highly dispersive channels in broadband wireless communications $[14,15]$. In both approaches, a cyclic prefix $(\mathrm{CP})$ is appended to each block for eliminating the interblock interference and converting, with respect to the useful part of the transmitted block, the linear convolution with the channel to circular. This allows low-complexity fast-Fourier transform-(FFT-) based receiver implementations. In recent years, SCFDE has become a powerful and an attractive link access method for the next-generation broadband wireless networks [16-18]. Because it is essentially a single-carrier system, SCFDE does not have some of the inherent problems of OFDM such as high PAPR. As a result, it has recently been receiving remarkable attention and has been adopted in the uplink of the Third Generation Partnership Project (3GPP) LongTerm Evolution (LTE) [19] system.

We show in this paper that the PAPR of ACO-SCFDE is quite less than that of ACO-OFDM and that its BER performance is better compared to ACO-OFDM when minimum mean square error (MMSE) detection is employed. The latter property is due to the inherent frequency diversity gain of SCFDE [20] and its low PAPR. Since the LED has limited linear range in its transfer characteristics, any values outside of that limited range will be clipped and distorted resulting in performance loss. We also propose in this paper two other schemes for generating real, positive signals with low PAPR for IM/DD optical DOW communications using SCFDE. The rest of the paper is organized as follows. In Section 2, we review the ACO-OFDM scheme. In Section 3, we present the proposed ACO-SCFDE. The two other newly proposed low PAPR schemes for optical communication using SCFDE which we call Repeat-and-Clipped Optical SCFDE (RCO-SCFDE) and Decomposed Quadrature Optical SCFDE (DQO-SCFDE) are presented in Sections 4 and 5, respectively followed by an analysis of the PAPR issues for DOW in Section 4. Performance analyses are presented in Section 7 followed by the conclusion in Section 8 .

Notations. Bold upper (lower) letters denote matrices (column vectors); $(\cdot)^{\mathcal{T}}$ and $(\cdot)^{\mathscr{H}}$ denote transpose and conjugate transpose (Hermitian), respectively. Throughout the paper, lower cases and upper, are used to represent time domain and frequency domain signals, respectively; $\star$ and $\odot$ represent linear and circular convolution, respectively; $\mathbf{I}_{N}$ denotes the identity matrix of size $N ; \mathbf{0}_{M \times N}$ denotes an all-zero matrix 
with size $M \times N$. For a complex number $a, \mathcal{R}_{e}(a)$ and $\ell_{m}(a)$ represent the real and imaginary part of $a$, respectively; for an $N \times 1$ vector $\mathbf{A},[\mathbf{A}(k)]_{k=0}^{N-1} \triangleq[A(0), A(1), \ldots, A(N-1)]^{T}$ and $\mathbf{A}^{*}$ is the vector of the conjugate of $\mathbf{A}$, that is, $\mathbf{A}^{*} \triangleq$ $\left[A^{*}(0), A^{*}(1), \ldots, A^{*}(N-1)\right]^{T}$.

\section{Review of Asymmetrically Clipped Optical OFDM (ACO-OFDM)}

The block diagram of a DOW communication system using ACO-OFDM is shown in Figure 2(a). The information stream is first parsed into a block of $N$ complex data symbols denoted by $\mathbf{S}=\left[S_{0}, S_{1}, \ldots, S_{N-1}\right]^{T}$, where the symbols are drawn from constellations such as QPSK, 16-QAM, or 64QAM with average electrical power $\mathrm{E}\left[\left|S_{k}\right|^{2}\right]=P_{s}$. These complex symbols are then mapped onto the following $4 N \times 1$ vector:

$$
\mathbf{X}=\left[0, S_{0}, 0, S_{1}, \ldots, 0, S_{N-1}, 0, S_{N-1}^{*}, 0, S_{N-2}^{*}, \ldots, 0, S_{0}^{*}\right]^{T} .
$$

Note that the average power of the block $\mathrm{X}$ is given by $E\left[\left|X_{k}\right|^{2}\right]=P_{s} / 2$. An $4 N$-point IFFT is then taken to construct the time domain signal $\mathbf{x}=\left[x_{0}, x_{1}, \ldots, x_{4 N-1}\right]^{T}$. A cyclic prefix is added to $\mathbf{x}$ as shown in Figure 2(b). The CP turns the linear convolution with the channel into a circular one, avoiding intercarrier interference (ICI) as well as interblock interference (IBI). To make the transmitted signal unipolar, all the negative values are clipped to zero to form the signal vector of $\tilde{\mathbf{x}}=\left[\tilde{x}_{4 N-L}, \ldots, \tilde{x}_{4 N-1}, \tilde{x}_{0}, \tilde{x}_{1}, \ldots, \tilde{x}_{4 N-1}\right]^{T}$ whose components are

$$
\tilde{x}_{n}= \begin{cases}x_{n} & \text { if } x_{n}>0, \\ 0 & \text { if } x_{n} \leq 0 .\end{cases}
$$

Because only the odd subcarriers are used to carry the data symbols, it is proved in [7] that the time-domain signal has an antisymmetry which ensures that clipping will not distort the odd subcarriers, but only reduce their amplitude by a factor of 2 ; hence the average transmitted electrical power (before the LED driving DC bias) is given by $E\left[\left|\tilde{x}_{n}\right|^{2}\right]=P_{s} / 4$.

The intermodulation caused by clipping occurs only in the even subcarriers and does not affect the data-carrying odd subcarriers. Note that the use of only odd subcarriers together with the Hermitian symmetry constraint cause only $N$ independent complex symbols to be transmitted out of the $4 N$ point IFFT. That is, the time domain signal $\mathbf{x}$ has a length of $4 N$ sample periods for $N$ input data symbols. The ACO-OFDM signal is then transmitted wirelessly via a light source (LED) through a diffuse optical channel and received by a photodetector. The received signal before the analog-todigital converter is given by

$$
\tilde{\mathbf{y}}=\tilde{\mathbf{x}} \star \mathbf{h}+\widetilde{\mathbf{w}},
$$

where $\mathbf{h}=[h(0), h(1), \ldots, h(L-1)]^{T}$ is the $L$-path impulse response of the optical channel, $\widetilde{\mathbf{x}}$ is the optical intensity of the transmitted signal block with the CP appended ( $\mathbf{x}$ is the transmitted block without the $\mathrm{CP}$ ), and $\widetilde{\mathbf{w}}$ is additive white Gaussian noise (AWGN) at the receiver. DOW links are subject to intense ambient light that gives rise to a highrate, signal-independent shot noise, which can be modeled as white and Gaussian [1]. When such ambient light is absent, the dominant noise is preamplifier thermal noise, which is Gaussian. Thus, we can model the noise as AWGN. Note that because the noise is added in the electrical domain, the received signal $\tilde{\mathbf{y}}$ can be negative as well as positive. So unlike the transmitted signal, the received signal is bipolar instead of unipolar. The CP is then removed to yield

$$
\mathbf{y}=\mathbf{x} \odot \mathbf{h}+\mathbf{w},
$$

where $\mathbf{w}$ is the noise vector without the CP. The linear convolution is turned into a circular one through the use of the CP $[21,22]$. To demodulate the signal, an $4 N$-point FFT is taken to access the frequency domain symbols

$$
\mathrm{Y}=\Lambda \mathrm{X}+\mathrm{W},
$$

where $\Lambda$ is a $4 N \times 4 N$ diagonal matrix whose diagonal is the $4 N$-point FFT of $\mathbf{h}$ and $\mathbf{W}$ is the $4 N$-point FFT of $\mathbf{w}$. The odd subcarriers are extracted from $\mathrm{Y}$ to yield

$$
\mathbf{Y}_{o}=\Lambda_{o} \overline{\mathbf{S}}+\mathbf{W}_{o}
$$

where

$$
\overline{\mathbf{S}}=\frac{1}{2}\left[S_{0}, S_{1}, \ldots, S_{N-1}, S_{N-1}^{*}, S_{N-2}^{*}, \ldots, S_{0}^{*}\right]^{T},
$$

$\mathbf{Y}_{o}$ and $\mathbf{W}_{o}$ are the vectors composed of the odd elements of $\mathbf{Y}$ and $\mathbf{W}$, respectively. The factor $1 / 2$ is due to the fact that the clipping caused the amplitude of each of the (odd) datacarrying subcarriers to be exactly half of its original value [7]. Similarly, $\boldsymbol{\Lambda}_{o}$ is a $2 N \times 2 N$ diagonal matrix whose diagonal contains the odd elements of the diagonal of $\boldsymbol{\Lambda}$.

To mitigate the effects of the channel, minimum-meansquare-error (MMSE) or zero-forcing (ZF) equalization can be used on $\mathbf{Y}_{o}$ to obtain an estimate for $\overline{\mathbf{S}}$ as follows:

$$
\hat{\overline{\mathbf{S}}}=\left(\boldsymbol{\Lambda}_{o}^{\mathcal{H}} \boldsymbol{\Lambda}_{o}+\left(\frac{\alpha}{\mathrm{SNR}}\right) \mathbf{I}_{2 N}\right)^{-1} \boldsymbol{\Lambda}_{o}^{\mathcal{H}} \mathbf{Y}_{o},
$$

where $\alpha=1$ for MMSE and $\alpha=0$ for ZF receivers and SNR is the electrical power of the transmitted symbol divided by the power of the electrical noise at the receiver. Due to the Hermitian symmetry condition, the symbols of $\boldsymbol{S}$ are repeated in $\overline{\mathbf{S}}$; hence we can add them after conjugation of the second half as follows:

$$
\widehat{\mathbf{S}}=[\hat{\widehat{\mathbf{S}}}(k)]_{k=0}^{N-1}+\left[\hat{\widehat{\mathbf{S}}}^{*}(2 N-1-k)\right]_{k=0}^{N-1} .
$$

Hard or soft detection is then made on the symbol of $\hat{\mathbf{S}}$. The extraction of odd subcarriers along with the equalization and the regrouping process of (9) are represented by the "Demapping" block in Figure 2.

The spectral efficiency (we define the spectral efficiency to be the number of modulated subcarriers over the total 


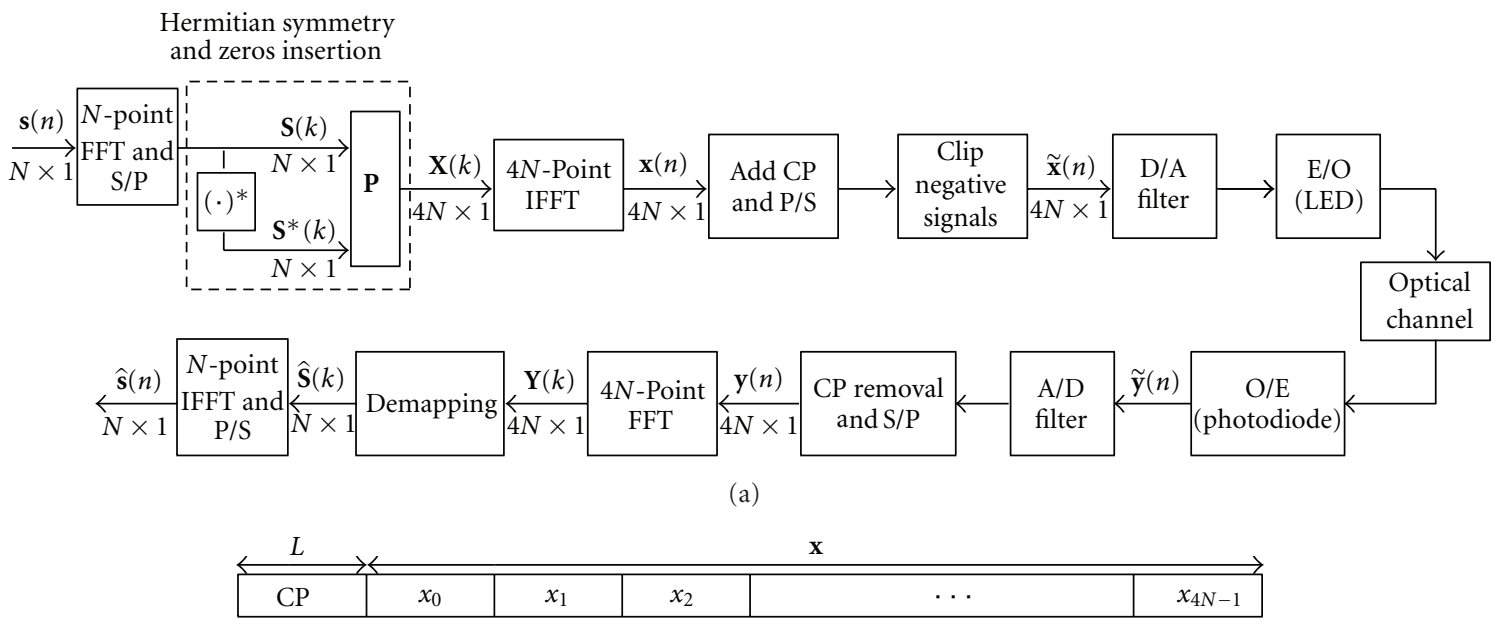

(b)

FIgURE 3: (a) ACO-SCFDE transmitter and receiver configuration. (b) ACO-SCFDE symbol after cyclic extension.

number of time-domain samples) of ACO-OFDM is given by

$$
\varepsilon_{\mathrm{ACO}}=\frac{N}{4 N+L}
$$

and is plotted in Figures 9 and 8 as a function of the number of subcarriers $N$ and channel delay spread where it is compared with other schemes.

To avoid the PAPR problem (which is examined later in this paper) of OFDM in DOW channels, a new modulation for optical communication using SCFDE is investigated in this paper. First we apply ACO-OFDM to SCFDE which we denote by ACO-SCFDE. We show that the latter exhibits better PAPR. We also show that the other proposed two modulation schemes for optical communication, called repetition and clipped optical SCFDE (RCO-SCFDE) and decomposed quadrature optical SCFDE (DQO-SCFDE), exhibit lower PAPR. Based on this fact, they are preferable for DOW communication where LED nonlinearity can affect the system performance.

\section{Asymmetrically Clipped Optical SCFDE (ACO-SCFDE)}

In this section, we apply asymmetrically clipped optical modulation to SCFDE to achieve ACO-SCFDE with low PAPR. SCFDE in its original form [14] cannot directly be applied to DOW with IM/DD. This is because the transmitted signal has to be real and positive while baseband SCFDE signals are generally complex and bipolar. In fact, ACO and DC-biased are two ways to obtain real positive signals from complex constellation symbols such as QPSK and M-QAM considered in this paper. As it was shown in [7] that ACO-OFDM is more power efficient than DCbiased OFDM, therefore in this paper, we focus on ACO which we applied to SCFDE and compare it with ACOOFDM. In ACO-SCFDE, an FFT and IFFT are used at the transmitter and the receiver. The additional complexity of the extra FFT at the transmitter, which is needed to obtain the Hermitian constraint on the frequency domain symbols, is offset by the fact that in SCFDE, the PAPR is reduced and better BER performance can be achieved when the signal is sent through a nonlinear LED. Let the $N$ input complex data symbols be denoted by the block $\mathbf{s}=$ $\left[s_{0}, s_{1}, \ldots, s_{N-1}\right]^{T}$ with average electrical power $E\left[\left|s_{n}\right|^{2}\right]=$ $P_{s}$. In order to achieve the Hermitian constraint, we first perform, at the transmitter, an $N$-point FFT on $\mathbf{s}$ to produce the frequency domain vector $S=\left[S_{0}, S_{1}, \ldots, S_{N-1}\right]^{T}$ with average power $E\left[\left|S_{k}\right|^{2}\right]=P_{s}$. As in ACO-OFDM, we map each of the $N$ symbols of $\mathbf{S}$ to $2 N$ Hermitian symmetric symbols and add zeroes to form the $4 N \times 1$ vector $\mathbf{X}=$ $\left[0, S_{0}, 0, S_{1}, \ldots, 0, S_{N-1}, 0, S_{N-1}^{*}, 0, S_{N-2}^{*}, \ldots, 0, S_{0}^{*}\right]^{T}$.

Due to the structure of $\mathbf{X}$ (zeros in the even locations), only the odd subcarriers carry data symbols. Next an $4 \mathrm{~N}$ point IFFT is used to obtain the time domain signal denoted by $\mathbf{x}=\left[x_{0}, x_{1}, \ldots, x_{4 N-1}\right]^{T}$. A CP is then added to $\mathrm{x}$ to yield $\tilde{\mathbf{x}}$ and the negative values are clipped to zero as in ACOOFDM. Hence, in ACO-SCFDE, the average transmitted electrical power (before the LED DC bias) is also given by $E\left[|\tilde{x}|^{2}\right]=P_{s} / 4$. The block diagram of this ACO-SCFDE scheme is shown in Figure 3(a) and the ACO-SCFDE symbol structure is shown in Figure 3(b). As will be seen later, the main advantage of ACO-SCFDE over ACO-OFDM is its lower PAPR. At the receiver, after removing the CP, an $4 \mathrm{~N}$ point FFT is applied. The odd subcarriers are then extracted exactly as in ACO-OFDM to yield the same equation as in (6) and the frequency domain symbol block $\mathbf{S}$ is estimated as in (9). After that, $\hat{S}$ is transformed back into the time domain to yield $\widehat{\mathbf{s}}=\mathbf{F}_{N}^{\mathscr{H}} \widehat{\mathbf{S}}$ where $\mathbf{F}_{N}^{\mathcal{H}}$ is the IFFT matrix. A hard or soft detection is made on $\hat{\mathbf{s}}$. The spectral efficiency of ACOSCFDE is the same as ACO-OFDM. The main difference between ACO-SCFDE and ACO-OFDM schemes is the addition of the $N$-point FFT and IFFT at the transmitter and receiver, respectively. The addition of an FFT and IFFT at the 


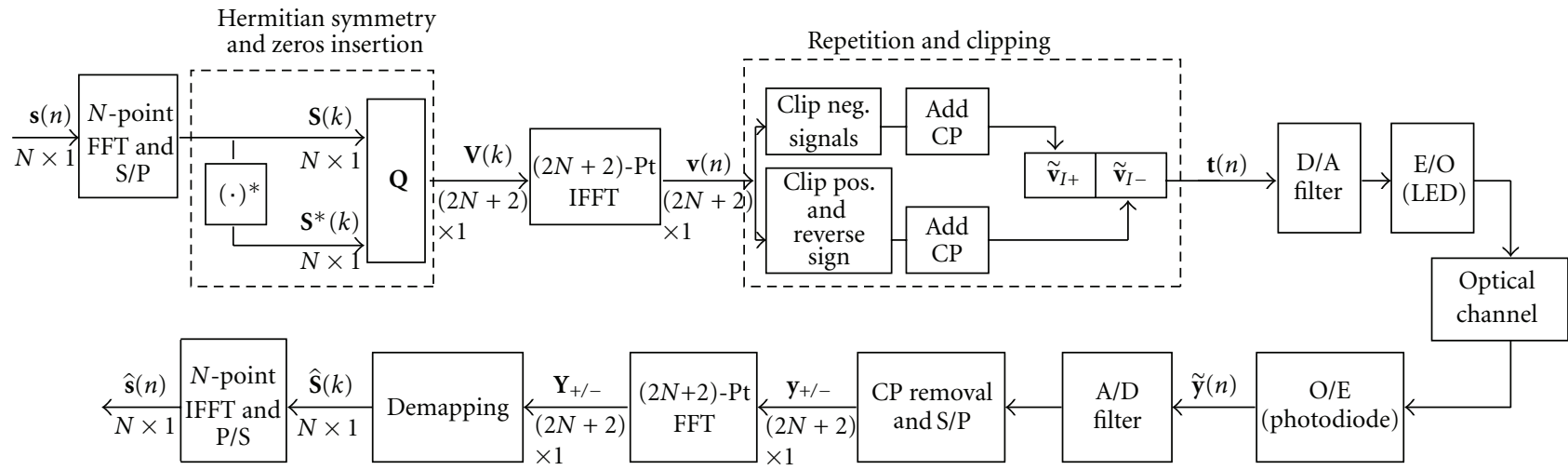

(a)

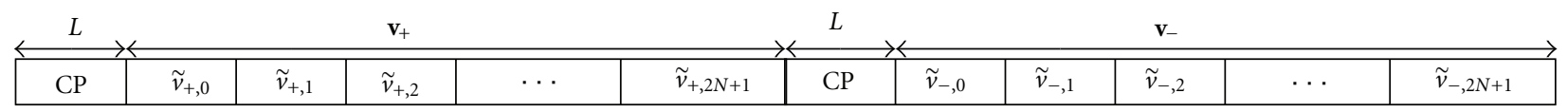

(b)

FIGURE 4: (a) RCO-SCFDE transmitter and receiver configuration. (b) RCO-SCFDE symbol after cyclic extension.

transmitter results in a single carrier transmission instead of multicarrier and hence reduction of the PAPR as shown in Figure 7.

\section{Repetition and Clipping Optical SCFDE (RCO-SCFDE)}

One drawback of the ACO-SCFDE or ACO-OFDM schemes is that only half of the subcarriers are used to carry data and the rest are set to zero. In another new scheme which we proposed in this section, called repetition and clipping optical SCFDE (RCO-SCFDE), only two subcarriers are set to zero, that is, do not carry data. The $N$ input complex data symbols $\mathbf{s}=\left[s_{0}, s_{1}, \ldots, s_{N-1}\right]^{T}$ with $E\left[\left|s_{n}\right|^{2}\right]=P_{s}$ are first transformed into the frequency domain to yield $N$ complex symbols which we denote by the block $\mathbf{S}=\left[S_{0}, S_{1}, \ldots, S_{N-1}\right]^{T}$ with $E\left[\left|S_{k}\right|^{2}\right]=P_{s}$. The Hermitian symmetry condition is achieved by forming the $(2 N+2) \times 1$ frequency domain vector

$$
\mathbf{V}=\left[0, S_{0}, S_{1}, \ldots, S_{N-1}, 0, S_{N-1}^{*}, S_{N-2}^{*}, \ldots, S_{0}^{*}\right]^{T}
$$

Note that the average power of $\mathbf{V}$ is $E\left[\left|V_{k}\right|^{2}\right] \approx P_{s}$. The block $\mathbf{V}$ is applied to a $(2 N+2)$-point IFFT (In implementing RCO-SCFDE, one should choose $N=2^{k}-1$, ( $k$ being an integer) such that $2 N+2$ is a power of 2 to reduce the complexity of IFFT.) to transform it back to the time domain vector $\mathbf{v}=\left[v_{0}, v_{1}, \ldots, v_{2 N+1}\right]^{T}$ with average electrical power $E\left[\left|v_{n}\right|^{2}\right] \approx P_{s}$. From the hermitian symmetry construction of (11), it is easily shown that the vector $\mathbf{v}$ is real. The block $\mathbf{v}$ is then repeated and clipped to yield the $(4 N+4) \times 1$ vector $\left[\mathbf{v}_{+}^{T} ; \mathbf{v}_{-}^{T}\right]^{T}$ as follows.

(i) In the first half of the repeated block, that is, in $\mathbf{v}_{+}$, the negative symbols of $\mathbf{v}$ are clipped to zeros. (ii) In the second half of the repeated block, that is, in $\mathbf{v}_{-}$, the positive symbols of $\mathbf{v}$ are clipped to zeros.

That is,

$$
\begin{aligned}
& v_{+, n}= \begin{cases}v_{n} & \text { if } v_{n}>0, \\
0 & \text { if } v_{n} \leq 0,\end{cases} \\
& v_{-, n}= \begin{cases}0 & \text { if } v_{n} \geq 0, \\
-v_{n} & \text { if } v_{n}<0,\end{cases}
\end{aligned}
$$

where $v_{+, n}$ and $v_{-, n}$ represent the $n$th $(n=0,1, \ldots, 2 N+1)$ element of $\mathbf{v}_{+}$and $\mathbf{v}_{-}$, respectively. A CP of length $L$ is then added to $\mathbf{v}_{+}$and $\mathbf{v}_{-}$to yield $\tilde{\mathbf{v}}_{+}$and $\tilde{\mathbf{v}}_{-}$, respectively. Note that the average electrical power of the block $\left[\mathbf{v}_{+}^{T} ; \mathbf{v}_{-}^{T}\right]^{T}$ is given by $P_{s} / 2$. The transmitted block is then denoted by the $(4 N+4+2 L) \times 1$ vector $\mathbf{t}=\sqrt{1 / 2}\left[\tilde{\mathbf{v}}_{+}^{T}, \tilde{\mathbf{v}}_{-}^{T}\right]^{T}$. The factor $\sqrt{1 / 2}$ is added to make the average transmitted electrical power the same as in the ACO-OFDM and ACO-SCFDE case, that is, $P_{s} / 4$. For notation simplicity, the normalizing factor $\sqrt{1 / 2}$ will be ignored in the following equations but will be taken into consideration in the simulation results. The block diagram of RCO-SCFDE is depicted in Figure 4(a) and the RCO-SCFDE is shown in Figure 4(b). The transmitted signal in this scheme is of length $4 N+4+2 L$ while it is $4 N+L$ in the ACO-SCFDE or ACO-OFDM case. That is there is then a slight bandwidth loss of $L+4$ symbols in this scheme. We note from (12) that

$$
\mathbf{v}=\mathbf{v}_{+}-\mathbf{v}_{-},
$$

and that the transmitted block $\mathbf{t}$ is composed of real positive signals. The received signal is given by

$$
\tilde{\mathbf{y}}=\mathbf{t} \star \mathbf{h}+\tilde{\mathbf{w}} \text {. }
$$




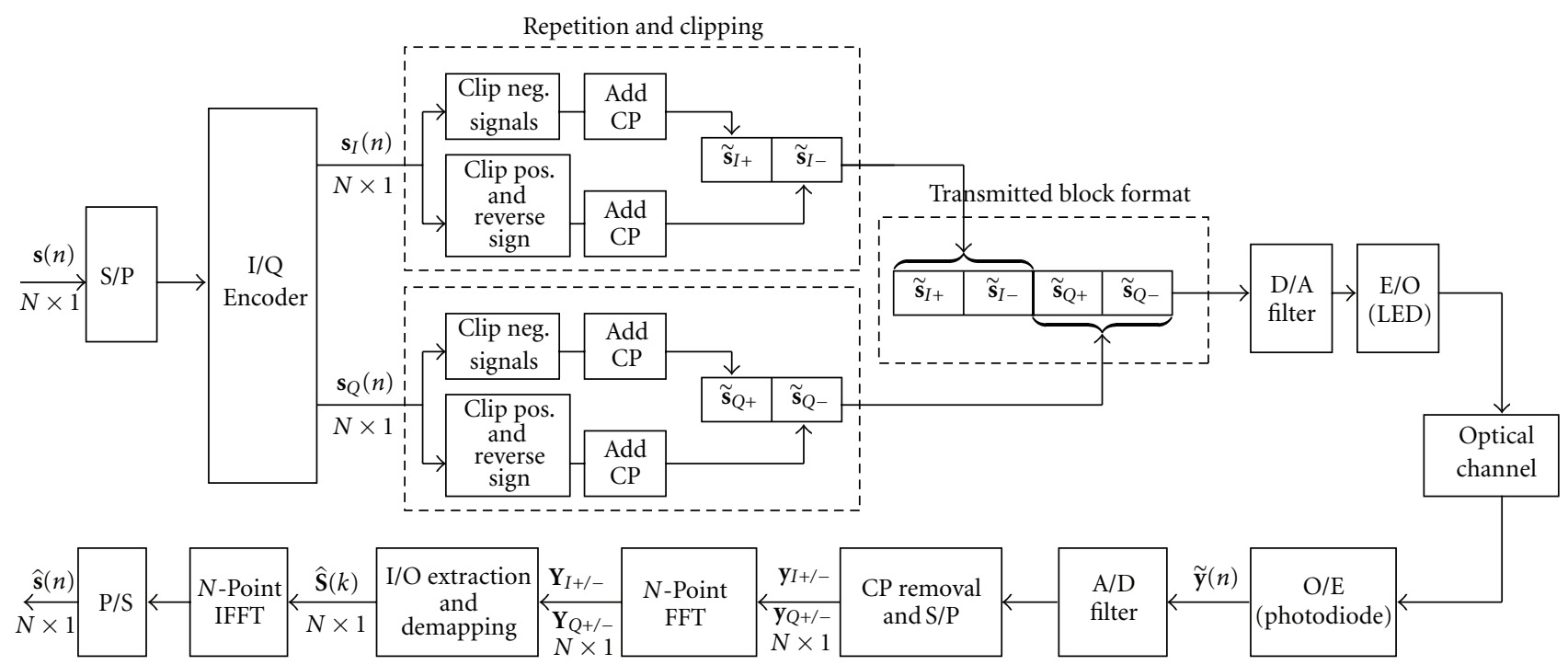

(a)

\begin{tabular}{|c|c|c|c|c|c|c|c|}
\hline$L$ & $N$ & $L$ & $N$ & $L$ & $N$ & $L$ & $N$ \\
\hline $\mathrm{CP}$ & $\mathbf{s}_{I+}$ & CP & $\mathbf{s}_{I-}$ & CP & $\mathbf{s}_{Q+}$ & CP & $\mathbf{s}_{Q-}$ \\
\hline
\end{tabular}

(b)

FIGURE 5: (a) DQO-SCFDE transmitter and receiver configuration. (b) DQO-SCFDE symbol after cyclic extension.

After removing the CP's, and using the fact that the CP makes linear convolution behave like cyclic convolution $[21,22]$, the received blocks corresponding to the first and second parts of t, (i.e., $\tilde{\mathbf{v}}_{+}$and $\left.\tilde{\mathbf{v}}_{-}\right)$are, respectively, given by the $(2 N+2) \times 1$ blocks $\mathbf{y}_{+}$and $\mathbf{y}_{-}$as follows

$$
\begin{aligned}
& \mathbf{y}_{+}=\mathbf{v}_{+} \odot \mathbf{h}+\mathbf{w}_{+}, \\
& \mathbf{y}_{-}=\mathbf{v}_{-} \odot \mathbf{h}+\mathbf{w}_{-},
\end{aligned}
$$

where $\mathbf{w}_{+}$and $\mathbf{w}_{-}$are the AWGN at the receiver. An $(2 N+2)$ point FFT is then taken separately on $\mathbf{y}_{+}$and $\mathbf{y}_{-}$to yield

$$
\begin{aligned}
& \mathbf{Y}_{+}=\Lambda^{\prime} \mathbf{V}_{+}+\mathbf{W}_{+}, \\
& \mathbf{Y}_{-}=\boldsymbol{\Lambda}^{\prime} \mathbf{V}_{-}+\mathbf{W}_{-},
\end{aligned}
$$

where $\mathbf{V}_{+}, \mathbf{V}_{-}, \mathbf{W}_{+}$, and $\mathbf{W}_{-}$, are the $(2 N+2)$-point FFT of $\mathbf{v}_{+}, \mathbf{v}_{-}, \mathbf{w}_{+}, \mathbf{w}_{-}$, respectively. $\boldsymbol{\Lambda}^{\prime}$ is a $(2 N+2) \times(2 N+2)$ diagonal matrix whose diagonal elements are the $(2 N+2)$ point FFT of $\mathbf{h}$.

The MMSE or ZF equalizer applied to $\mathbf{Y}_{+}$and $\mathbf{Y}_{-}$yield

$$
\begin{aligned}
& \hat{\mathbf{V}}_{+}=\left(\boldsymbol{\Lambda}^{\prime \mathcal{H}} \boldsymbol{\Lambda}^{\prime}+\left(\frac{1}{\mathrm{SNR}}\right) \mathbf{I}_{2 N+2}\right)^{-1} \boldsymbol{\Lambda}^{\prime \mathcal{H}} \mathbf{Y}_{+} \\
& \hat{\mathbf{V}}_{-}=\left(\boldsymbol{\Lambda}^{\prime \mathcal{H}} \boldsymbol{\Lambda}^{\prime}+\left(\frac{1}{\mathrm{SNR}}\right) \mathbf{I}_{2 N+2}\right)^{-1} \boldsymbol{\Lambda}^{\prime} \mathbf{H}_{-} .
\end{aligned}
$$

From (13), we note that $\mathbf{V}=\mathbf{V}_{+}-\mathbf{V}_{-}$, hence we can form the estimated vector

$$
\hat{\mathbf{V}}=\hat{\mathbf{V}}_{+}-\hat{\mathbf{V}}_{-} \text {. }
$$

Using (11), the frequency domain transmitted symbols $\mathbf{S}$ are then estimated as

$$
\widehat{\mathbf{S}}=[\hat{\mathbf{V}}(k)]_{k=1}^{N}+\left[\hat{\mathbf{V}}^{*}(2 N+2-k)\right]_{k=1}^{N},
$$

where the subcarriers 0 and $N+1$ were dropped since they do not carry any data. We then obtain the time domain signal by the taking an $N$-point IFFT of $\widehat{\mathbf{S}}$ followed by a hard or soft detection. The spectral efficiency of RCO-SCFDE is given by

$$
\varepsilon_{\mathrm{RCO}}=\frac{N}{4 N+2 L+4}
$$

and depicted in Figure 9 as a function of the number of subcarrier $\mathrm{N}$ and channel delay spread L. Figure 9 also demonstrates its efficiency compared to other schemes.

The main advantages of RCO-SCFDE are

(i) in ACO-SCFDE and ACO-OFDM, only half of the electrical power is used on the odd frequency, datacarrying subcarriers. The other half is used on the even subcarriers which are discarded at the receiver. RCO-SCFDE does not have this disadvantage;

(ii) the PAPR of RCO-SCFDE is lower than that ACOOFDM and is plotted in Figure 7;

(iii) the size of the IFFT at the transmitter is $2 \mathrm{~N}+2$ while it is $4 \mathrm{~N}$ for ACO-SCFDE and ACO-OFDM. 


\section{Decomposed Quadrature Optical SCFDE (DQO-SCFDE)}

With this scheme, a different technique than the Hermitian symmetry constraint is used to generate the real positive symbols needed for intensity modulated direct detection (IM/DD) optical communication. In the previous schemes, after modulating subcarriers with Hermitian symmetry, one must use an IFFT to transform the signal into the time domain before transmission. The use of an IFFT increases the PAPR of the transmitted signal. In this new scheme which we call Decomposed Quadrature Optical SCFDE (DQOSCFDE), the real (in-phase) and imaginary (quadrature) part of the complex modulated symbols are transmitted separately as follows. Let the input $N$ complex data symbols be denoted by the block $\mathbf{s}=\left[s_{0}, s_{1}, \ldots, s_{N-1}\right]^{T}$ with $E\left[\left|s_{n}\right|^{2}\right]=P_{s}$ and let $\boldsymbol{s}_{I}=\left[\mathcal{R}_{e}\left(s_{0}\right), \mathcal{R}_{e}\left(s_{1}\right), \ldots, \mathcal{R}_{e}\left(s_{N-1}\right)\right]$ and $\mathbf{s}_{Q}=\left[\ell_{m}\left(s_{0}\right), \ell_{m}\left(s_{1}\right), \ldots, \ell_{m}\left(s_{N-1}\right)\right]$ the vector of the real (inphase) and imaginary (quadrature) part of $s$, respectively. As in RCO-SCFDE case, we form the vectors $\boldsymbol{s}_{I_{+}}, \boldsymbol{s}_{I_{-}}, \mathbf{s}_{Q_{+}}$, and $\mathbf{s}_{Q_{-}}$, as follows:

$$
\begin{gathered}
s_{I_{+}}(n)= \begin{cases}s_{I}(n) & \text { if } s_{I}(n)>0, \\
0 & \text { if } s_{I}(n) \leq 0,\end{cases} \\
s_{I_{-}}(n)= \begin{cases}0 & \text { if } s_{I}(n) \geq 0, \\
-s_{I}(n,) & \text { if } s_{I}(n)<0 .\end{cases}
\end{gathered}
$$

$\mathbf{s}_{Q_{+}}$and $\mathbf{s}_{Q_{-}}$are similarly defined. A CP is added to each subblock to yield the $(N+L) \times 1$ vectors $\widetilde{\mathbf{s}}_{I, i}$ and $\widetilde{\mathbf{s}}_{Q, i}$, and the following $4(N+L)$ real and positive symbol block $\tilde{\mathbf{x}}$ is transmitted

$$
\widetilde{\mathbf{x}}=\left[\widetilde{\mathbf{s}}_{I_{+}}, \widetilde{\mathbf{s}}_{I_{-}}, \widetilde{\mathbf{s}}_{Q_{+}}, \widetilde{\mathbf{s}}_{Q_{-}}\right]^{T} .
$$

Note that we have

$$
\begin{gathered}
\mathbf{s}_{I}=\mathbf{s}_{I_{+}}-\mathbf{s}_{I_{-}}, \\
\mathbf{s}_{Q}=\mathbf{s}_{Q_{+}}-\mathbf{s}_{Q_{-}} .
\end{gathered}
$$

One can easily show that the average transmitted electrical power in this case is also given by $P_{s} / 4$. The block diagram of DQO-SCFDE is shown in Figure 5. The received signal is given by

$$
\tilde{\mathbf{y}}=\tilde{\mathbf{x}} \star \mathbf{h}+\widetilde{\mathbf{w}} .
$$

After removing the CP's, the received subblock of length $N$ corresponding to the transmitted in-phase $\boldsymbol{s}_{I_{+}}$and $\mathbf{s}_{I_{-}}$are given by

$$
\begin{aligned}
& \mathbf{y}_{I_{+}}=\mathbf{s}_{I_{+}} \odot \mathbf{h}+\mathbf{w}_{I_{+}}, \\
& \mathbf{y}_{I_{-}}=\mathbf{s}_{I_{-}} \odot \mathbf{h}+\mathbf{w}_{I_{-}},
\end{aligned}
$$

and the received subblock of length $N$ corresponding to the transmitted quadrature $\boldsymbol{s}_{Q_{+}}$and $\boldsymbol{s}_{Q_{-}}$are given by

$$
\begin{aligned}
& \mathbf{y}_{Q_{+}}=\mathbf{s}_{Q_{+}} \star \mathbf{h}+\mathbf{w}_{Q_{+}}, \\
& \mathbf{y}_{Q_{-}}=\mathbf{s}_{Q_{-}} \star \mathbf{h}+\mathbf{w}_{Q_{-}} .
\end{aligned}
$$

The $N \times 1$ vectors $\mathbf{w}_{I+}\left(\mathbf{w}_{I-}\right)$ and $\mathbf{w}_{Q_{+}}\left(\mathbf{w}_{Q_{-}}\right)$are the AWGN associated with the received in-phase and quadrature subblocks, respectively. An N-point FFT is then performed for each received $N$ symbols subblock to yield

$$
\begin{aligned}
\mathbf{Y}_{I_{+}} & =\Lambda \mathbf{S}_{I_{+}}+\mathbf{W}_{I_{+}}, \\
\mathbf{Y}_{I_{-}} & =\Lambda \mathbf{S}_{I_{-}}+\mathbf{W}_{I_{-}} .
\end{aligned}
$$

$\mathbf{Y}_{Q_{+}}$and $\mathbf{Y}_{Q_{+}}$are similarly defined where $\boldsymbol{\Lambda}_{N}$ is an $(N \times N)$ diagonal matrix whose diagonal is the $N$-point FFT of $\mathbf{h}$. The MMSE or ZF equalizer yields

$$
\begin{aligned}
& \hat{\mathbf{S}}_{I_{+}}=\left(\boldsymbol{\Lambda}_{N}^{\mathcal{H}} \boldsymbol{\Lambda}_{N}+\left(\frac{\alpha}{\mathrm{SNR}}\right) \mathbf{I}_{N}\right)^{-1} \boldsymbol{\Lambda}_{N}^{\mathcal{H}} \mathbf{Y}_{I_{+}}, \\
& \hat{\mathbf{S}}_{I_{-}}=\left(\boldsymbol{\Lambda}_{N}^{\mathcal{H}} \boldsymbol{\Lambda}_{N}+\left(\frac{\alpha}{\mathrm{SNR}}\right) \mathbf{I}_{N}\right)^{-1} \boldsymbol{\Lambda}_{n}^{\mathcal{H}} \mathbf{Y}_{I_{-}} .
\end{aligned}
$$

$\widehat{\mathbf{S}}_{Q_{+}}$and $\widehat{\mathbf{S}}_{Q_{-}}$are similarly defined. Using (23), we form the estimated vector

$$
\begin{gathered}
\widehat{\mathbf{S}}_{I}=\widehat{\mathbf{S}}_{I_{+}}-\widehat{\mathbf{S}}_{I_{-}}, \\
\hat{\mathbf{S}}_{Q}=\widehat{\mathbf{S}}_{Q_{+}}-\widehat{\mathbf{S}}_{Q_{-}} .
\end{gathered}
$$

The frequency domain transmitted symbols $\mathbf{S}$ are then estimated as

$$
\widehat{\mathbf{S}}=\widehat{\mathbf{S}}_{I}+j \widehat{\mathbf{S}}_{Q},
$$

where $j=\sqrt{-1}$. We then obtain the time domain signal by the taking an $N$-point IFFT of $\widehat{\boldsymbol{S}}$ followed by a hard or soft detection. The spectral efficiency of DQO-SCFDE is given by

$$
\varepsilon_{\mathrm{DQO}}=\frac{N}{4(N+L)}
$$

and is depicted in Figure 9 as a function of the number of subcarrier $N$ and channel delay spread $L$ where it is compared with other schemes. Also the PAPR is given in Figure 7.

\section{Peak-to-Average Power Ratio Issues}

Like conventional OFDM systems, high PAPR can be a serious penalty in optical OFDM systems $[23,24]$. In radio frequency (RF) communications, the power amplifier is the main source of nonlinearity while in DOW communications, the LED is the nonlinear device that limits the performance of optical OFDM. The nonlinear characteristic of an LED imposes limitations on the performance of indoor DOW systems when using intensity modulation with both ACOOFDM and DC-biased OFDM [9] because of their high PAPR. The sensitivity of OFDM to nonlinearities is also presented in $[6,25-27]$. The PAPR is usually presented in terms of a Complementary Cumulative Distribution Function (CCDF) which is the probability that PAPR is higher than a certain PAPR value $\mathrm{PAPR}_{0}$, that is, $\operatorname{Pr}\{\mathrm{PAPR}>$ $\left.\mathrm{PAPR}_{0}\right\}$. In Figure 7, the CCDF is calculated by Monte Carlo simulation for QPSK, 16 QAM, and 64 QAM modulation constellations. CCDF of PAPR for ACO-OFDM as well as 
the proposed ACO-SCFDE, RCO-SCFDE, and DQO-SCFDE are evaluated and compared. It can be seen that the PAPR of ACO-OFDM is the highest while DQO-SCFDE exhibits the lowest PAPR.

Several techniques have been proposed to reduce the PAPR of OFDM signal, such as filtering, clipping, coding, partial transmission sequences (PTS), and selected mapping (SLM) [28-33]. Whereas filtering has a disadvantage due to the noise and exogenous disturbance generated by nonlinear operations [28], the coding technique is confined by its high complexity and efficiency degradation [31]. Probability techniques such as PTS and SLM also have the disadvantage of high complexity computation $[32,33]$. The proposed SCFDE schemes for DOW in this paper exhibit lower PAPR with low complexity. DQO-SCFDE has the lowest PAPR and lowest complexity; it should then be considered as a strong candidate in future DOW communication with IM/DD.

\section{Performance Analysis}

In this paper, simulations have been conducted using the commercial high power IR LED (OSRAM, SFH 4230) [25] whose transfer characteristic is shown in Figure 6. A polynomial of the sixth degree has been shown to model this transfer function using a least-square curve fitting approach [25]. Figure 6 shows the relation between the forward voltage across the LED and the current through it. Any input voltage less than $1.3 \mathrm{~V}$ or more than $2.1 \mathrm{~V}$ is clipped. From the LED characteristic depicted, it can be seen that the LED transfer function is linear only between $1.6 \mathrm{~V}$ and $1.85 \mathrm{~V}$. If the input voltage has high dynamic range, the peak voltage will be distorted or clipped which will result in performance loss. The optical power is proportional to the LED forward current that is, $P_{\mathrm{opt}}=\zeta x^{\prime}(t)$ where $x^{\prime}(t)$ represent the LED forward current and we have assumed that $\zeta=1$ [34]. In the simulations, a DC bias of $1.6 \mathrm{~V}$ has been used to drive the LED into the linear region of the LED transfer function.

7.1. Complexity Analysis. In this subsection, we compare the computational complexity of the three newly proposed modulation techniques ACO-SCFDE, RCO-SCFDE, DQOSCFDE and with that of ACO-OFDM. First, we note that all the transceivers take as input a block of $N$ independent complex data symbols to be transmitted using different techniques through a diffuse DOW channel. The main difference lies in how the transmitted block at the input of the LED is formed. For ACO-OFDM, the computational complexity is mainly due to the $4 N$-point FFT at the transmitter and the $4 \mathrm{~N}$-point IFFT at the receiver. So the complexity of ACO-OFDM is of order $\mathcal{O}\left(8 N \log _{2}(4 N)\right)$. The complexity of ACO-SCFDE is the same as ACO-OFDM plus the additional $N$-point FFT and $N$-point IFFT at the transmitter and receiver, respectively, hence ACO-SCFDE complexity is of order $\mathcal{O}\left(8 N \log _{2}(4 N)+2 N \log _{2}(N)\right)$. In RCO-SCFDE, a $(2 N+2)$-point FFT is taken at the transmitter and $(2 N+2)$-point IFFT is taken at the receiver twice (once for each block $\mathbf{y}_{+}$and $\mathbf{y}_{-}$) and as in ACO-SCFDE, RCO-SCFDE also has the additional complexity of $N$-point

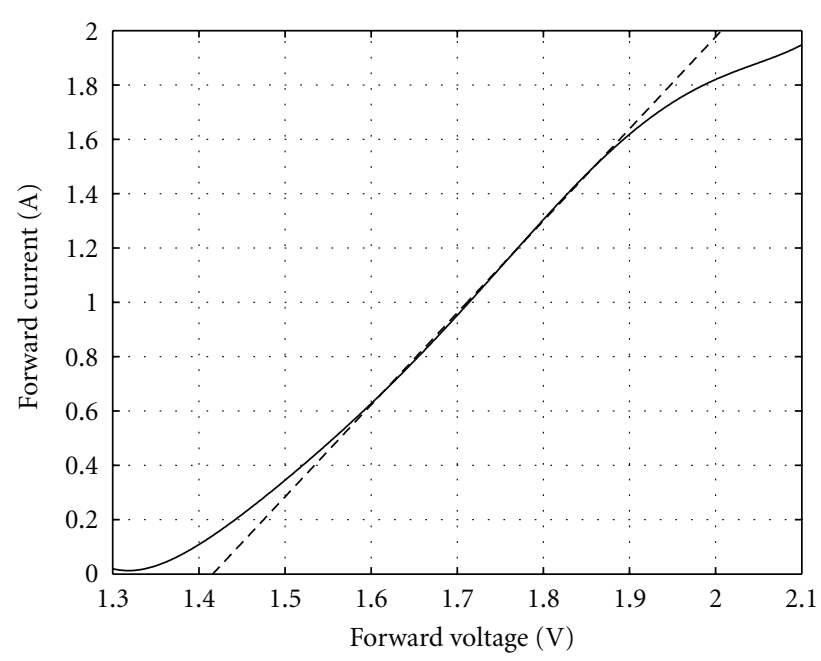

FIgURE 6: The LED transfer characteristics of the OSRAM, SFH 4230 showing the forward voltage and forward current relation. The dashed line shows the function that corresponds to the linear region of the LED transfer response.

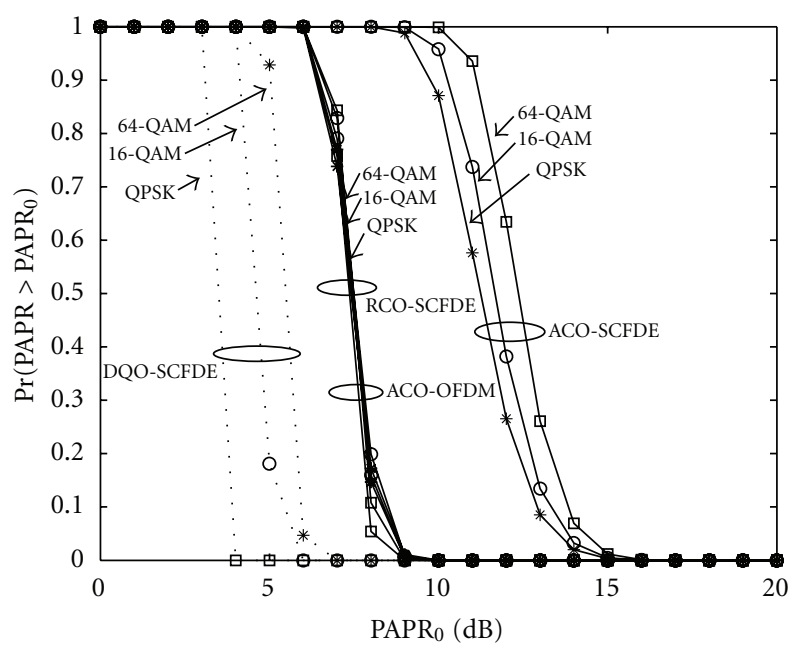

FIGURE 7: CCDF of PAPR comparison of ACO-OFDM, ACOSCFDE, RCO-SCFDE, and DQO-SCFDE0.

FFT and $N$-point IFFT at the transmitter and receiver, respectively. Since $N$ is a power of $2,2 N+2$ is not a power of 2. But if we choose in RCO-SCFDE $N$ as $2^{k}-1$ for any integer $k, 2 N+2$ will be a power of 2 and the complexity of RCO-SCFDE can be given as of order $\mathcal{O}\left(3(2 N+2) \log _{2}(2 N+\right.$ $\left.2)+2 N \log _{2}(N)\right)$. In DQO-SCFDE, there is only an $N$-point FFT performed at the receiver four times and an $N$-point IFFT taken once to transform the symbols into the time domain at the output. There is not a computational burden on the transmitter. The complexity of DQO-SCFDE is of the order of $\mathcal{O}\left(4 N \log _{2}(N)+N \log _{2}(N)\right)$. These complexities are summarized in Table 1 and plotted as a function of the input block size $N$ in Figure 10.

7.2. Simulation Results. This section displays simulation results for ACO-OFDM, ACO-SCFDE, RCO-SCFDE and, 


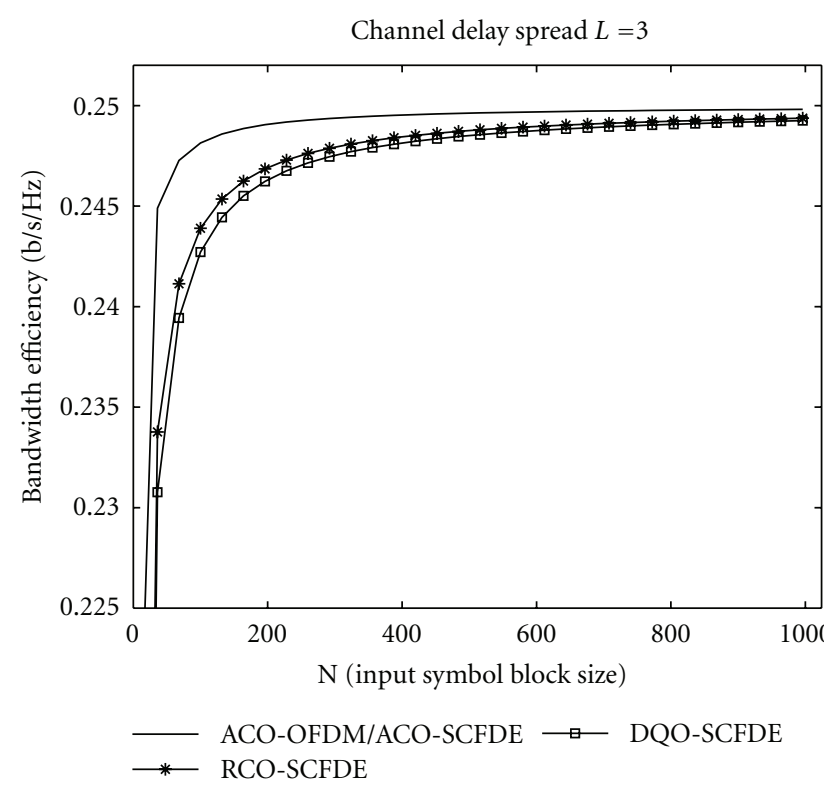

FIgURE 8: Bandwidth efficiency comparison for ACO-OFDM, ACO-SCFDE, RCO-SCFDE, and DQO-SCFDE with channel delay spread of $L=3$ sampling times.

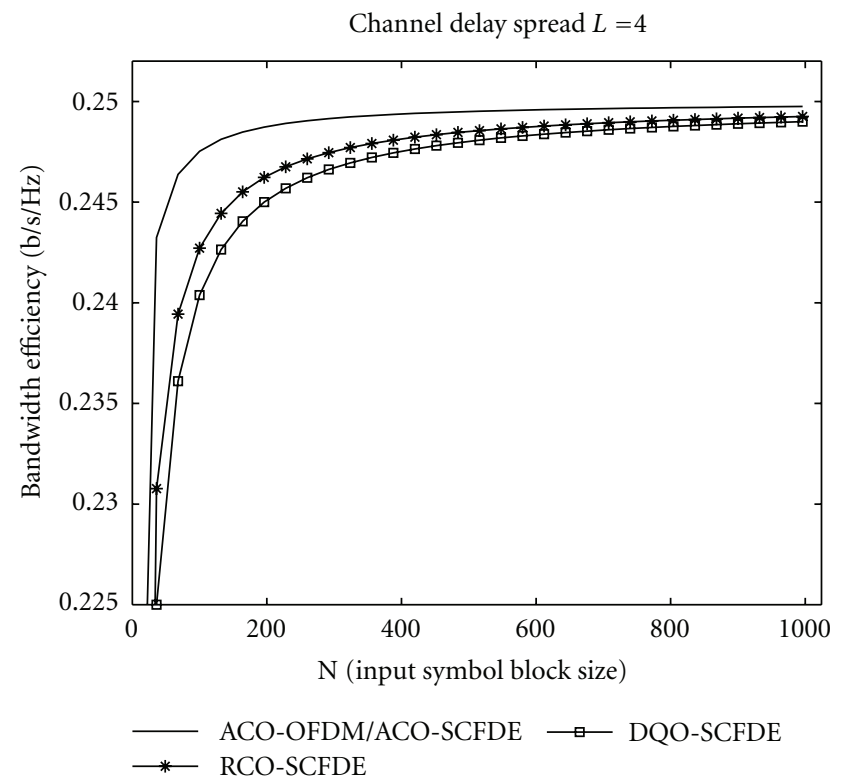

FIGURE 9: Bandwidth efficiency comparison for ACO-OFDM, ACO-SCFDE, RCO-SCFDE, and DQO-SCFDE with channel delay spread of $L=4$ sampling times.

DQO-SCFDE schemes with $N=64$ independent data symbols. QPSK, 16 QAM, and 64 QAM modulation constellations are used. We considered three different input symbol average power levels $P_{s}=0.1 \mathrm{~W}, 0.5 \mathrm{~W}$, and $1 \mathrm{~W}$ for QPSK and $P_{s}=0.01 \mathrm{~W}$ and $0.1 \mathrm{~W}$ for 16 QAM and 64 QAM. Hence the transmitted block average electrical powers at the input of the LED are, respectively, given by $P_{s} / 4=25 \mathrm{~mW}, 125 \mathrm{~mW}$, and $250 \mathrm{~mW}$ for QPSK and $P_{s} / 4=2.5 \mathrm{~mW}$ and $25 \mathrm{~mW}$ for $16 \mathrm{Q}$ AM and 64Q AM. A DC bias of $1.6 \mathrm{~V}$ is added to

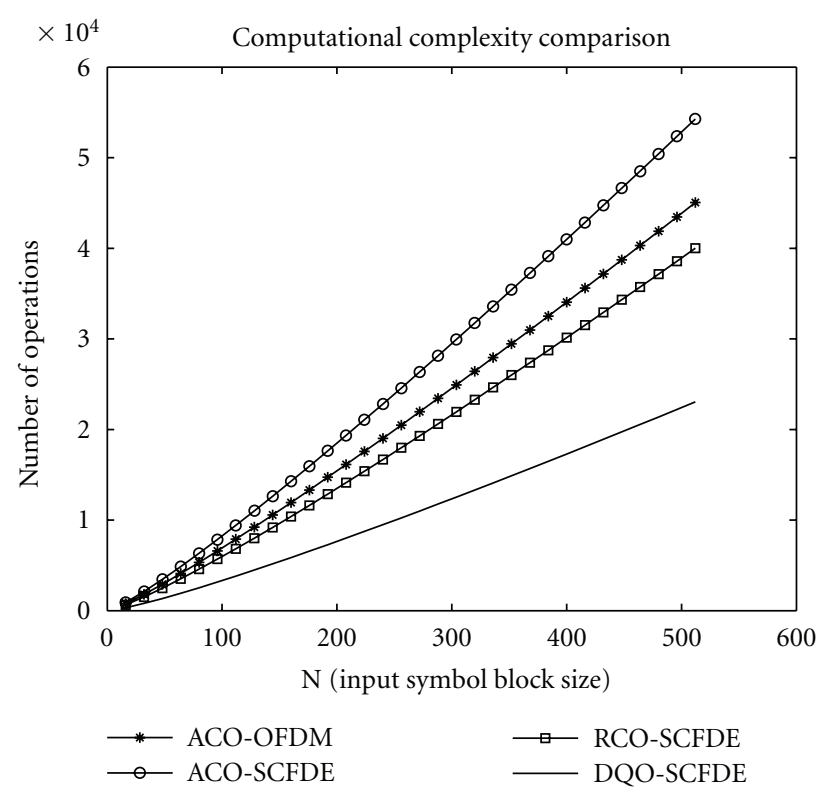

Figure 10: Computational comparison for ACO-OFDM, RCOSCFDE, and DQO-SCFDE.

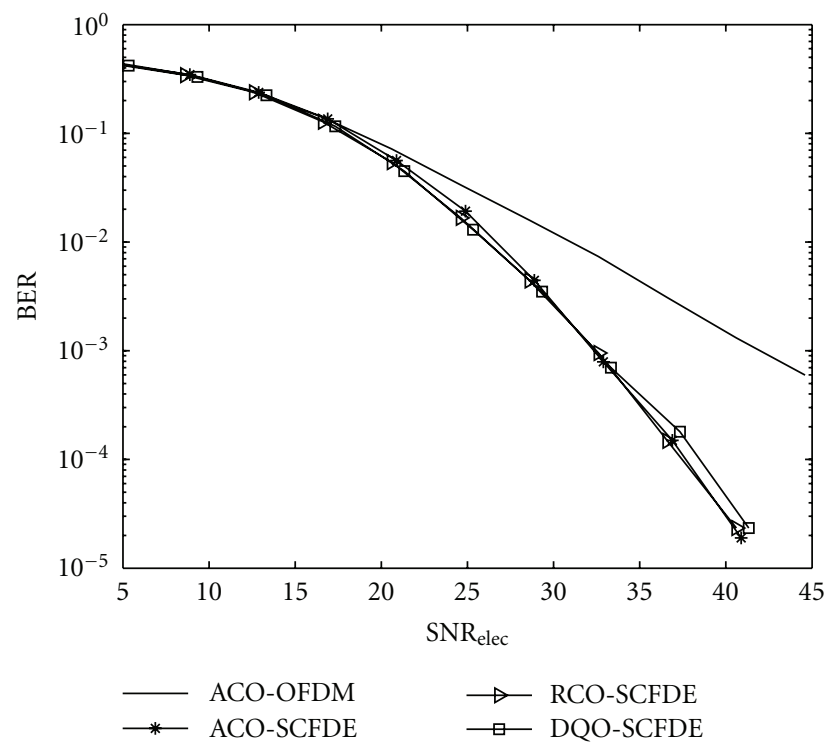

Figure 11: MMSE BER comparison of ACO-OFDM, ACO-SCFDE, RCO-SCFDE, and DQO-SCFDE with $N=64$, QPSK input symbols with power $0.1 \mathrm{~W}$ and $L=3$.

drive the LED in all schemes. The exponential power decay channel model is used with a maximum delay spread of $L=3$ sampling periods with real and positive taps [35] and the CP is set to $L$ symbols. The channel is assumed perfectly known at the receiver. MMSE and ZF frequency domain equalization are used to mitigate the effects of the channel.

We first compare the PAPR of all schemes as shown in Figure 7 from which we notice that DQO-SCFDE has the lowest PAPR while ACO-OFDM has the highest. Hence 


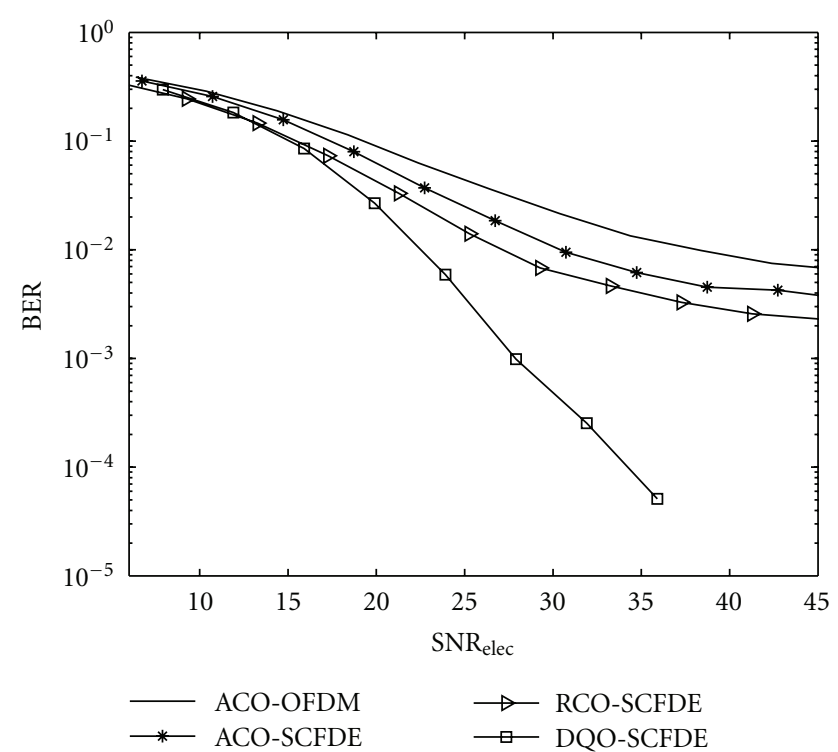

FIGURE 12: MMSE BER comparison of ACO-OFDM, ACO-SCFDE, RCO-SCFDE, and DQO-SCFDE with $N=64$, QPSK input symbols with average power $0.5 \mathrm{~W}, L=3$.

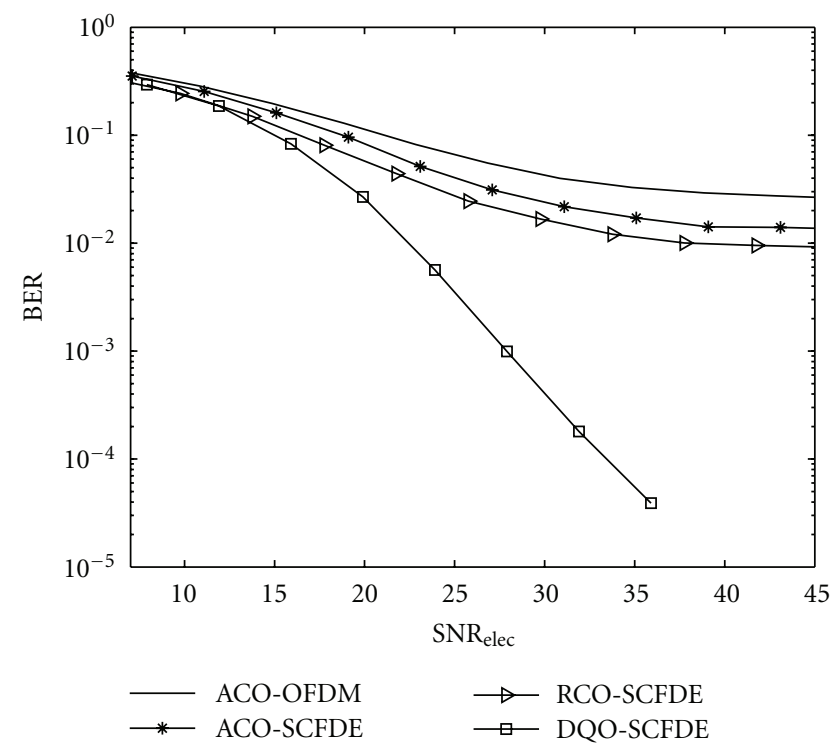

FIGURE 13: MMSE BER comparison of ACO-OFDM, ACO-SCFDE, RCO-SCFDE, and DQO-SCFDE with $N=64$, QPSK input symbols with power $1 \mathrm{~W}$ and $L=3$.

DQO-SCFDE is the preferable in terms of PAPR. Large PAPR signal affects the performance of the system as the linear range of the transfer function of the LED is limited. SCFDE uses single carrier, hence its PAPR is inherently lower than OFDM which uses multicarriers. One will then expect that the BER performance of the SCFDE schemes will be better. This will be clarified in the following BER performance analysis.

Next we compare the spectral efficiencies of the different schemes as plotted in Figures 9 and 8 for channel delay

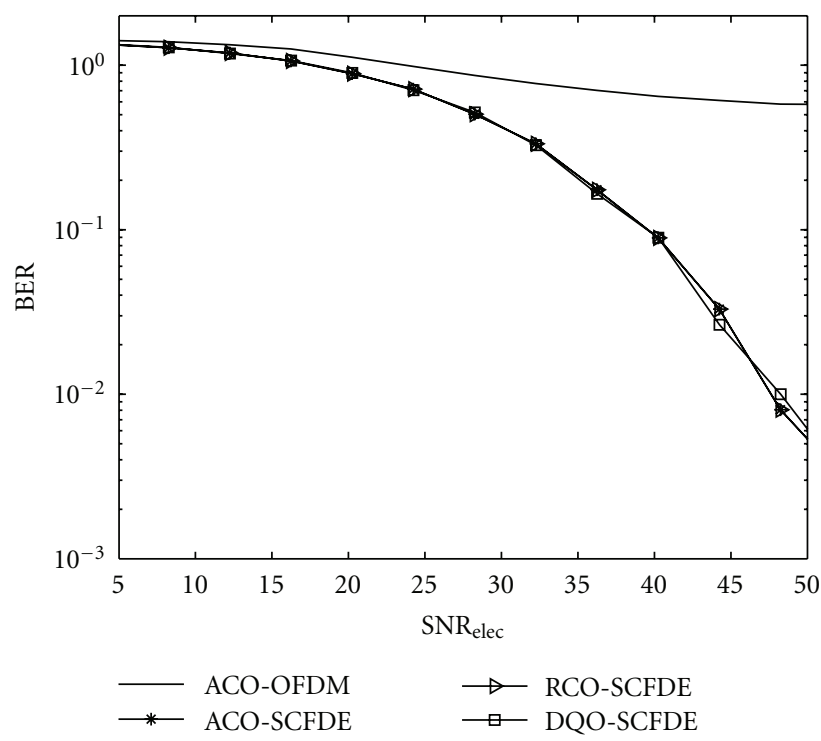

FIGURE 14: MMSE BER comparison of ACO-OFDM, ACO-SCFDE, RCO-SCFDE, and DQO-SCFDE with $N=64,16 \mathrm{QAM}$ input symbols with power $0.01 \mathrm{~W}, L=3$.

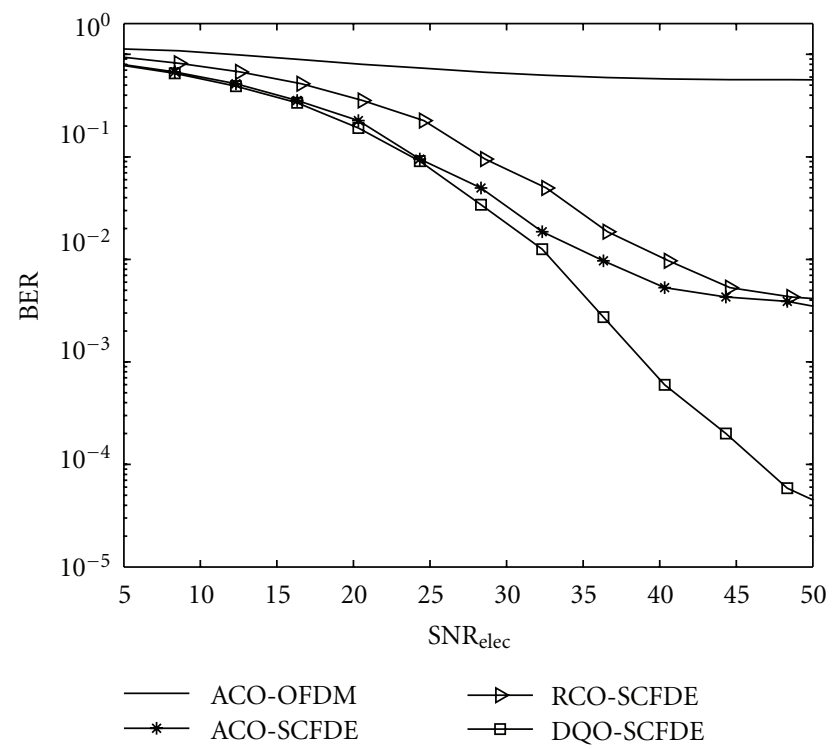

Figure 15: MMSE BER comparison of ACO-OFDM, ACO-SCFDE, RCO-SCFDE, and DQO-SCFDE with $N=64,16 \mathrm{QAM}$ input symbols with average power $0.1 \mathrm{~W}, L=3$.

spread of $L=3$ and $L=4$, respectively (For indoor DOW system, a maximum of 3 or 4 taps are sufficient to model the channel impulse response [36]). It can be seen that as the input block size $N$ is large, the bandwidth efficiencies are almost the same for all schemes. Hence if $N$ is large, the bandwidth loss experienced by RCO-SCFDE and DQOSCFDE is negligible.

Finally BER performances are analyzed. We have only plotted the results for the MMSE equalizer which are shown in Figures 11, 12, 13, 14, 15, 16, and 17. We first note the BER 


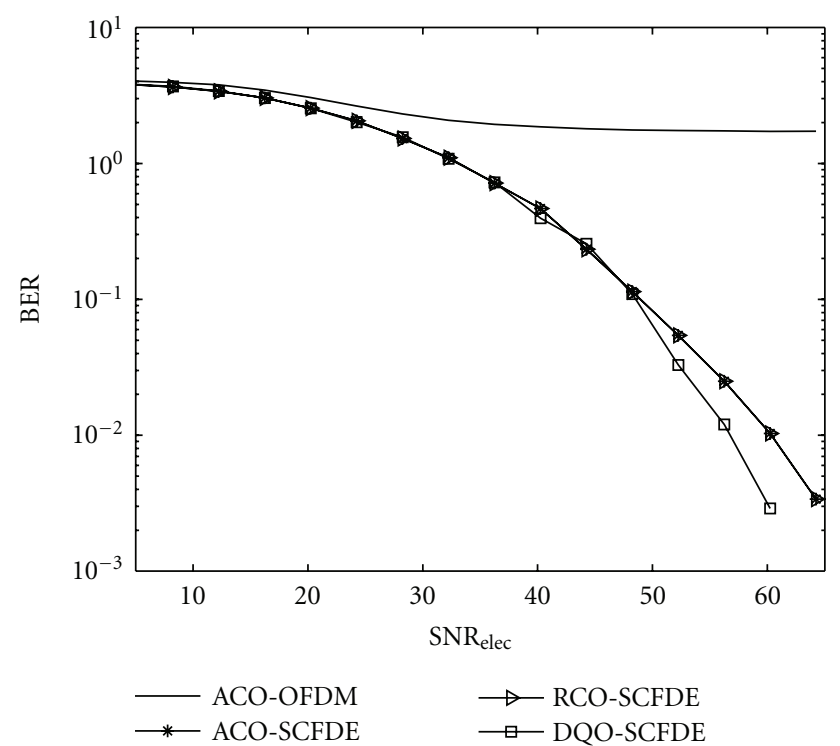

FIGURE 16: MMSE BER comparison of ACO-OFDM, ACO-SCFDE, RCO-SCFDE, and DQO-SCFDE with $N=64,64$ QAM input symbols with power $0.01 \mathrm{~W}, L=3$.

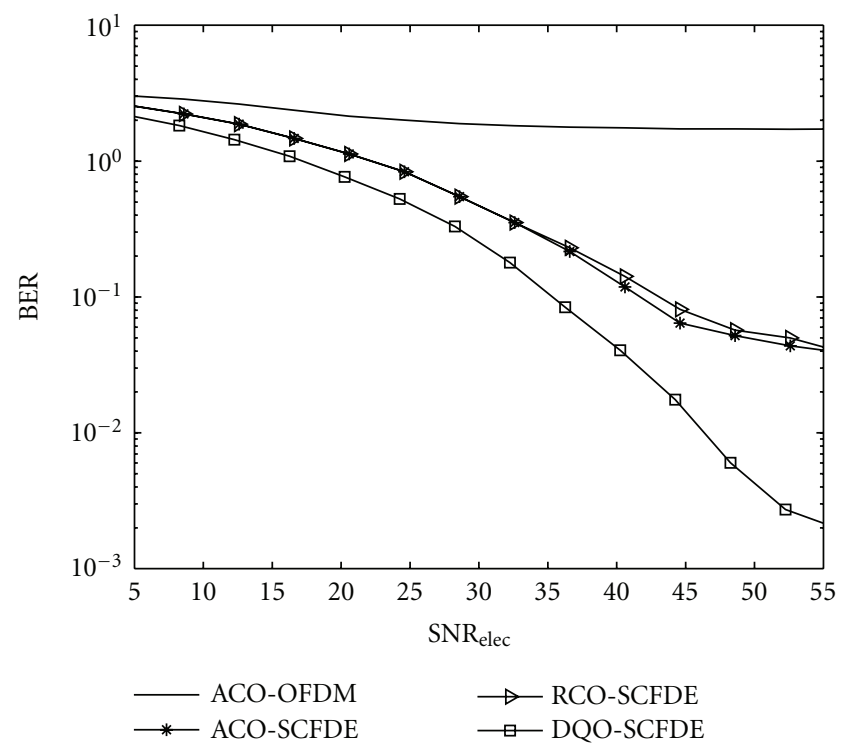

FIGURE 17: MMSE BER comparison of ACO-OFDM, ACO-SCFDE, RCO-SCFDE, and DQO-SCFDE with $N=64$, 64 QAM input symbols with average power $0.1 \mathrm{~W}, L=3$.

performance of the proposed SCFDE schemes when MMSE is used is always better than that of ACO-OFDM.

From Figures 11 to 13 , we have plotted the BER performance for QPSK modulation with input symbol average power of $P_{s}=0.1 \mathrm{~W}, 0.5 \mathrm{~W}$, and $1 \mathrm{~W}$. We note that when the input power is low, that is, $0.1 \mathrm{~W}$, the BER performances of the SCFDE schemes are all the same. This is because the PAPR is low and most of the signal values are within the linear range of the LED response. However, when the input power is higher, that is, $1 \mathrm{~W}$ in the QPSK case, DQO-SCFDE
TABLE 1: Computational complexity comparison of the four modulation techniques.

\begin{tabular}{lc}
\hline Schemes & Complexity \\
\hline ACO-OFDM & $\mathcal{O}\left(8 N \log _{2}(4 N)\right)$ \\
ACO-SCFDE & $\mathcal{O}\left(8 N \log _{2}(4 N)+2 N \log _{2}(N)\right)$ \\
RCO-SCFDE & $\mathcal{O}\left[3(2 N+2) \log _{2}(2 N+2)+2 N \log _{2}(N)\right]$ \\
DQO-SCFDE & $\mathcal{O}\left(5 N \log _{2}(N)\right)$ \\
\hline
\end{tabular}

scheme performance is much better. This result confirms the PAPR results shown in Figure 7, that is, DQO-SCFDE has quite lower PAPR than the other schemes. DQO-SCFDE signal amplitudes are lower which results in less clipping and distortion. Also, we note that all SCFDE schemes outperform ACO-OFDM in all cases especially when the input power is increasing. The bad performance of ACO-OFDM is due the fact that the PAPR is higher and hence many signal values are outside the linear range of the LED response which creates signal distortion which in turns causes the performance loss. When the input symbol power is low, that is, $0.01 \mathrm{~W}$, ACO-OFDM performance is better than for $0.1 \mathrm{~mW}$ but its performance is still worst that SCFDE schemes. This is because with SCFDE, a spectral null in the channel negatively affects all the symbols in a block [37] which is not the case for MMSE equalization as was also shown in performance study of SCFDE in [37]. Moreover, SCFDE has an inherent diversity gain due to the use of the IFFT at the receiver which causes peaks and the nulls of the frequency response to spread across several data values.

When larger size constellations are used, that is, 16 QAM and 64QAM, ACO-OFDM performance has the worst performance and reliable communication cannot happen as can be seen in Figures 14 to 17. This is again due the fact that for the larger constellation size, the PAPR of ACO-OFDM is higher and hence substantial signal clipping and distortion occur that affect the system performance. We also note that, DQO-SCFDE performance is the best in all case especially when the input symbol power is increased. This is because, the PAPR of DQO-SCFDE is so low that by increasing the input symbol power, most of the signal values fall within the linear range of the LED response, hence no or less signal distortion occurs. When the input symbol power is lower, that is, $0.01 \mathrm{~W}$, the performances of all SCFDE schemes are almost the same due to their lower PAPR. For an input signal power of $1 \mathrm{~W}$ for QPSK and $0.1 \mathrm{~W}$ for 16 QAM and 64 QAM, DQO-SCFDE performs better than ACO-SCFDE due to its lower PAPR. These simulation results show the effectiveness of the SCFDE schemes when the nonlinearity of the LED is considered. In general, if nonlinearity is not considered, increasing signal power decreases BER. But when the nonlinearity of the LED is considered, we want a system that has good BER for low signal power. DQOSCFDE has the best performance among all the schemes. However, increasing the signal power is more detrimental for ACO-OFDM due to its higher PAPR. High peaks in the signal are clipped or distorted which results in the BER floor. 


\section{Conclusion}

In this paper, we present three new modulation techniques for diffuse optical wireless communications with IM/DD. The first applies asymmetrically clipped optical (ACO) principles to SCFDE which we called ACO-SCFDE. The others, namely, RCO-SCFDE and DQO-SCFDE, use the newly introduced technique of repetition and clipping. It was shown through the use of simulation that these new techniques exhibit lower PAPR and better BER performance in a multipath channel. The spectral efficiency of these techniques is almost the same when the symbol block size is sufficiently large. ACO-SCFDE is a direct application of ACO-OFDM using SCFDE modulation instead of OFDM. The former requires FFT and IFFT at the transmitter and receiver but has lower PAPR than ACO-OFDM and better BER performance. RCO-SCFDE and DQO-SCFDE are other two new methods for generating real positive signal needed for transmission over the optical channel. RCO-SCFDE has the same PAPR as ACO-SCFDE but lower computational complexity. DQO-SCFDE has the lowest PAPR, lower computational complexity, and exhibits better BER performances. For this particular reason, we believe that DQO-SCFDE is the most attractive choice for transmitting real positive signal over an optical channel.

\section{References}

[1] J. M. Kahn and J. R. Barry, "Wireless infrared communications," Proceedings of the IEEE, vol. 85, no. 2, pp. 265-298, 1997.

[2] B. Wilson and Z. Ghassemlooy, "Pulse time modulation techniques for optical communications: a review," IEE Proceedings J: Optoelectronics, vol. 140, no. 6, pp. 346-357, 1993.

[3] W. Shieh, X. Yi, Y. Ma, and Q. Yang, "Coherent optical OFDM: has its time come?" Journal of Optical Networking, vol. 7, no. 3, pp. 234-255, 2008.

[4] J. B. Carruthers and J. M. Kahn, "Multiple-subcarrier modulation for nondirected wireless infrared communication," IEEE Journal on Selected Areas in Communications, vol. 14, no. 3, pp. 538-546, 1996.

[5] O. González, R. Pérez-Jiménez, S. Rodríguez, J. Rabadán, and A. Ayala, "OFDM over indoor wireless optical channel," vol. 152, no. 4, pp. 199-204.

[6] H. Elgala, R. Mesleh, and H. Haas, "Indoor broadcasting via white LEDs and OFDM," IEEE Transactions on Consumer Electronics, vol. 55, no. 3, pp. 1127-1134, 2009.

[7] J. Armstrong and A. J. Lowery, "Power efficient optical OFDM," Electronics Letters, vol. 42, no. 6, pp. 370-372, 2006.

[8] J. Armstrong, "OFDM for optical communications," Journal of Lightwave Technology, vol. 27, no. 3, pp. 189-204, 2009.

[9] J. Armstrong and B. J. C. Schmidt, "Comparison of asymmetrically clipped optical OFDM and DC-biased optical OFDM in AWGN," vol. 12, no. 5, pp. 343-345.

[10] H. Elgala, R. Mesleh, and H. Haas, "A study of LED nonlinearity effects on optical wireless transmissionusing OFDM," in Proceedings of the 6th internationalconference on Wireless and Optical Communications Networks (WOCN '09), pp. 388-392, IEEE Press, Piscataway, NJ, USA, 2009.

[11] H. Elgala, R. Mesleh, and H. Haas, "Predistortion in optical wireless transmission using OFDM," in Proceedings of the 9th International Conference on Hybrid Intelligent Systems (HIS '09), vol. 2, pp. 184-189, August 2009.

[12] K. Ishihara, T. Kobayashi, R. Kudo et al., "Frequency-domain equalisation for optical transmission systems," Electronics Letters, vol. 44, no. 14, pp. 870-872, 2008.

[13] C.-C. Hsieh and D.-S. Shiu, "Single carrier modulation with frequency domain equalization for intensity modulationdirect detection channels with intersymbol interference," in Proceedings of the 17th IEEE International Symposium on Personal, Indoor and Mobile Radio Communications (PIMRC '06), September 2006.

[14] D. Falconer, S. L. Ariyavisitakul, A. Benyamin-Seeyar, and B. Eidson, "Frequency domain equalization for single-carrier broadband wireless systems," IEEE Communications Magazine, vol. 40, no. 4, pp. 58-66, 2002.

[15] H. Sari, G. Karam, and I. Jeanclaude, "Transmission techniques for digital terrestrial TV broadcasting," IEEE Communications Magazine, vol. 33, no. 2, pp. 100-109, 1995.

[16] H. Sari, G. Karam, and I. Jeanclaude, "Frequency-domain equalization of mobile radio and terrestrial broadcast channels," in Proceedings of the IEEE Global Telecommunications Conference (GLOBECOM '94), vol. 1, pp. 1-5, 1994.

[17] M. V. Clark, "Adaptive frequency-domain equalization and diversity combining for broadband wireless communications," IEEE Journal on Selected Areas in Communications, vol. 16, no. 8, pp. 1385-1395, 1998.

[18] A. Gusmao, R. Dinis, J. Conceicao, and N. Esteves, "Comparison of two modulation choices for broadband wireless communications," in Proceedings of the IEEE Vehicular Technology Conference (VTC '00), vol. 2, pp. 1300-1305, Tokyo, Japan, May 2000.

[19] H. Ekström, A. Furuskär, J. Karlsson et al., "Technical solutions for the 3G long-term evolution," IEEE Communications Magazine, vol. 44, no. 3, pp. 38-45, 2006.

[20] Y.-P. Lin and S.-M. Phoong, "BER minimized OFDM systems with channel independent precoders," IEEE Transactions on Signal Processing, vol. 51, no. 9, pp. 2369-2380, 2003.

[21] Z. Wang and G. B. Giannakis, "Wireless multicarrier communications: where Fourier meets Shannon," IEEE Signal Processing Magazine, vol. 17, no. 3, pp. 29-48, 2000.

[22] S. B. Weinstein and P. M. Ebert, "Data transmission by frequency-division multiplexing using the discrete Fourier transform," IEEE Transactions on Communications, vol. 19, no. 5, pp. 628-634, 1971.

[23] X. Liang, W. Li, W. Ma, and K. Wang, "A simple peak-toaverage power ratio reduction scheme for all optical orthogonal frequency division multiplexing systems with intensity modulation and direct detection," Optics Express, vol. 17, no. 18, pp. 15614-15622, 2009.

[24] H. Paul and K.-D. Kammeyer, "Linearization of transmitter and receiver nonliniearity in optical OFDM transmission," in Proceedings of the 7th International Workshopon Multi-Carrier Systems and Solutions, May 2009.

[25] H. Elgala, R. Mesleh, and H. Haas, "Practical considerations for indoorwireless optical system implementation using OFDM," in Proceedings of the IEEE 10th InternationalConference on Telecommunications (ConTEL '09), p. 810, Zagreb, Croatia, June 2009.

[26] H.-D. Han, J. Hu, and Z. Ding, "A bandwidth efficient design of IM/DD optical OFDM," in Proceedings of the Conference on Lasers and Electro-Optics and Conference on Quantum Electronics and Laser Science Conference (CLEO/QELS '09), pp. 1-2, June 2009. 
[27] Y. Tang, K.-P. Ho, and W. Shieh, "Coherent optical OFDM transmitter design employing predistortion," IEEE Photonics Technology Letters, vol. 20, no. 11, pp. 954-956, 2008.

[28] X. Li and L. J. Cimini Jr., "Effects of clipping and filtering on the performance of OFDM," IEEE Communications Letters, vol. 2, no. 5, pp. 131-133, 1998.

[29] M. Faulkner, "The effect of filtering on the performance of OFDM systems," IEEE Transactions on Vehicular Technology, vol. 49, no. 5, pp. 1877-1884, 2000.

[30] S.-K. Deng and M.-C. Lin, "Recursive clipping and filtering with bounded distortion for PAPR reduction," IEEE Transactions on Communications, vol. 55, no. 1, pp. 227-230, 2007.

[31] S. H. Han and J. H. Lee, "Modified selected mapping technique for PAPR reduction of coded OFDM signal," IEEE Transactions on Broadcasting, vol. 50, no. 3, pp. 335-341, 2004.

[32] S. H. Müller and J. B. Huber, "OFDM with reduced peakto-average power ratio by optimum combination of partial transmit sequences," Electronics Letters, vol. 33, no. 5, pp. 368369, 1997.

[33] R. W. Bäuml, R. F. H. Fischer, and J. B. Huber, "Reducing the peak-to-average power ratio of multicarrier modulation by selected mapping," Electronics Letters, vol. 32, no. 22, pp. 2056-2057, 1996.

[34] J. Armstrong, B. J. C. Schmidt, D. Kalra, H. A. Suraweera, and A. J. Lowery, "Performance of asymmetrically clipped optical OFDM in AWGN for an intensity modulated direct detection system," in Proceedings of the IEEE Global Telecommunications Conference (GLOBECOM '06), pp. 1-5, November 2006.

[35] J. B. Carruthers, "Modeling of nondirected wireless infrared channels," IEEE Transactions on Communications, vol. 45, no. 10, pp. 1260-1268, 1997.

[36] J. R. Barry, J. M. Kahn, W. J. Krause, E. A. Lee, and D. G. Messerchmitt, "Simulation of multipath impulse response for indoor wireless optical channels," IEEE Journal on Selected Areas in Communications, vol. 11, no. 3, pp. 367-379, 1993.

[37] Y.-P. Lin and S.-M. Phoong, "MMSE OFDM and prefixed single carrier systems: BER analysis," in Proceedings of the IEEE International Conference on Accoustics, Speech, and Signal Processing, vol. 4, pp. 229-232, April 2003. 